\title{
Caracterización clínico-epidemiológica de Traumatismo Craneoencefálico Severo Pediátrico en Hospital Nacional Mario Catarino Rivas 2016-2018
}

\author{
Clinical-epidemiological characterization of Severe Pediatric Brain injury at \\ Hospital Nacional Mario Catarino Rivas 2016-2018
}

Sofía Marlene Cardona Pineda*, Iván Estrada**, Raúl Anariba** Luisamaría Pineda**

\section{RESUMEN}

Antecedentes: Traumatismo craneoencefálico (TCE) se define como un intercambio brusco de energía mecánica causado por una fuerza externa que tiene como resultado una alteración a nivel anatómico-funcional del encéfalo y sus envolturas. Objetivo: Caracterizar clínica y epidemiológicamente los TCE severos en pacientes de 2-18 años, atendidos en Unidad de Cuidados Intensivos Pediátricos (UCIP) del Hospital Mario Catarino Rivas (HMCR) durante enero 2016 a mayo 2018. Pacientes y métodos: Estudio descriptivo transversal observacional. Universo de 809 pacientes. La muestra por conveniencia fue de $77(9.5 \%$, IC95\%), se incluyó pacientes de 2-18 años, con expediente clínico completo. Se excluyeron ingresos por causa no traumática, traslados a otros centros hospitalarios y pacientes con TCE leve-moderado. Las variables incluyeron datos sociodemográficos, características del traumatismo, manifestaciones clínicas, tratamiento en UCIP, complicaciones asociadas y condición de egreso. La información recolectada fue ingresada en una base de datos en Epiinfo versión 7.2.2.6, se realizó un análisis univariado. La información extraída de los expedientes se manejó confidencialmente. Resultados: El grupo etáreo más afectado fueron escolares $40.2 \%$ (31), el mecanismo de lesión más

*Médico Residente tercer año de Pediatría, UNAH-VS.

**Pediatra intensivista, Unidad de Cuidados Intensivos Pediátricos, HNMCR.

***Epidemióloga Pediatra, HMCR.

Dirigir correspondencia a: sof15cp@hotmail.com

Recibido: 30 de Enero $2019 \quad$ Aprobado: 15 de Agosto 2019 común accidente de tránsito $64.9 \%$ (50). El $97.4 \%$ (75) presentó pérdida del estado de consciencia, la región anatómica más afectada fue temporal en $52.0 \%$ (40). $45.9 \%$ iniciaron alimentación enteral antes de las $12 \mathrm{hrs}$, la prevalencia fue de $9.5 \%$ y la mortalidad del 10\%. Conclusión: Es importante identificar los mecanismos de trauma, para dar un manejo oportuno e intervenir de manera eficaz, con el fin de disminuir la morbimortalidad de estos.

\section{PALABRAS CLAVE}

Lesiones Traumáticas del Encéfalo, Escala de Coma de Glasgow, Cuidados Críticos, Pediatría.

\section{ABSTRACT}

Background: Cranioencephalic traumatism (CET) is defined as a sudden exchange of mechanical energy caused by an external force that results in an anatomical-functional alteration of the brain and its envelopes. Objective: To characterize clinically and epidemiologically the severe CET in the population 218 years attended in the HNMCR in the area of PICU during January 2016 to May 2018. Patients and methods: Observational cross-sectional descriptive study. Universe of 809 pediatric patients. A convenience sample size of $77(9.5 \%$, IC95\%) was estimated. Patients aged 2-18 years were included, with a complete clinical record. Non-traumatic admissions, transfers to other hospitals and patients with mild-moderate CET were excluded. The variables included sociodemographic data, trauma 
characteristics, clinical manifestations, PICU treatment, associated complications and discharge condition. The information collected was entered into a database, designed with the statistical package Epiinfo version 7.2.2.6 (CDC Atlanta), a univariate analysis was performed. Information extracted from the files was handled confidentially. Results: The most affected age group were schoolchildren $40.2 \%$ (31), the most common injury mechanism was traffic accident $64.9 \%(50)$. $97.4 \%$ (75) presented loss of consciousness, the most affected anatomical region was temporary in $52.0 \%$ (40). $45.9 \%$ started enteral feeding before 12 o'clock, prevalence was $9.5 \%$ and mortality $10 \%$. Conclusion: It is important to identify the mechanisms of trauma, to provide timely management and intervene effectively, in order to reduce the morbidity and mortality of these.

\section{KEYWORDS}

Brain Injuries, Traumatic, Critical Care, Glasgow Coma Scale, Pediatrics.

\section{INTRODUCCIÓN}

Los accidentes representan una importante causa de morbilidad - mortalidad a cualquier edad y la población pediátrica no es la excepción, así las lesiones en niños siguen siendo un problema de salud pública en todo el mundo. La Organización Mundial de la Salud (OMS) estima que mueren en todo el mundo, aproximadamente 100 niños cada hora a causa de lesiones, de las cuales el $90 \%$ son no intencionales. ${ }^{(1)}$ Se define como un intercambio brusco de energía mecánica causado por una fuerza externa que tiene como resultado una alteración a nivel anatómico y/o funcional (motora, sensorial y/o cognitiva) del encéfalo y sus envolturas, en forma precoz o tardía, permanente o transitoria. ${ }^{(2,3)}$

Según la OMS el TCE se ha convertido en un problema socioeconómico a nivel mundial. Por tanto, el panorama es estremece dor y preocupante ya que se estima que para el 2020 se convierta en una de las primeras causas de muerte y discapacidad en el mundo (Soto, 2013). EI TCE representa el $9 \%$ al $35 \%$ de muerte accidental en la niñez, la mayoría de los casos secundario a politraumatismos, de estos aproximadamente 60.000 de los casos al año producen muerte y cerca de 90.000 casos condiciones de discapacidad permanente. (3-7) Se ha documentado que $15.8 \%$ de los pacientes pediátricos requieren ser tratados en una unidad de cuidados intensivos (Guillen, 2013). EI TCE afecta la etapa aguda a nivel vascular, neuronal, glial, y cardiorrespiratorio, alterando funciones motoras, sensoriales y cognitivas que se definen como secuelas en meses 0 años. ${ }^{(4,8-10)}$

Existen factores que favorecen que el paciente pediátrico sea víctima de lesiones accidentales, por ejemplo, dada su falta de experiencia, la imprudencia propia de su edad, y en el caso del paciente lactante y preescolar, su capacidad exploradora. El espíritu temerario del adolescente y la necesidad psicológica de aceptación social frecuentemente lo impulsan a tomar actitudes de riesgo. Existen otros elementos que predisponen al paciente pediátrico al momento de sufrir lesiones por accidentes en los diferentes escenarios, factores que los hacen diferentes a los adultos y que son determinantes para el pronóstico de los mismos, por ejemplo el diámetro y el peso de la cabeza, la estatura, la gran cantidad de agua corporal total que hace más elásticos ligamentos importantes en columna, la laxitud de los huesos largos, menor rigidez en la pared abdominal y la flexibilidad de las estructuras de protección en la cabeza de los pacientes. ${ }^{(11-14)}$ Cada año, alrededor de 10 millones de niños en todo el mundo requieren hospitalización a consecuencia de lesiones no intencionales; de ellas, el 95\% ocurren en países de ingresos económicos 
intermedios o bajos. ${ }^{(1)}$ Finalmente el tratamiento y la evaluación del trauma pediátrico requieren no solo de un amplio conocimiento sobre la anatomo-fisiología, sino sobre los mecanismos y posibles órganos involucrados en cada uno de estos; la edad de presentación más frecuente, distribución por género, región geográfica donde más ocurren, así como todas las características sociodemográficas y clínicas que se relacionan con el trauma, para así poder ofrecer un completo e integral manejo, con el objetivo de ofrecer un estratégico y adecuado plan de promoción y prevención, optimización del recurso físico y humano dentro de las instituciones, así como el principal aporte que como institución dará al país, que en conjunto permita crear datos epidemiológicos para la región y formar así, parte de las casuísticas nacionales e internacionales. Por esta razón el objetivo principal de este estudio fue caracterizar clínica y epidemiológicamente traumatismos craneoencefálicos severos en la población pediátrica de 2-18 años atendidos en la Unidad de Cuidados Intensivos Pediátricos del Hospital Nacional Mario Catarino Rivas, durante enero 2016 a mayo 2018.

\section{PACIENTES Y MÉTODOS}

Se realizó un estudio descriptivo transversal sobre los pacientes pediátricos ingresados a la Unidad de Cuidados Intensivos Pediátricos del Hospital Nacional Mario Catarino Rivas, San Pedro Sula Honduras, en el período del mes de enero 2016 al mes de mayo de 2018. En este período ingresaron 809 pacientes en edad pediátrica. De este total, cumplían con diagnóstico de TCE severo 123 (15.2\%). Para describir las características de los pacientes pediátricos con TCE severo, se estimó un tamaño de muestra de 77 casos (IC95\%), el muestreo fue no probabilístico. La fuente de información la constituyeron los expedientes clínicos y el libro de registros de la Unidad de
Cuidados Intensivos Pediátricos.

Se incluyó pacientes pediátricos de 2 a 18 años, con expediente clínico completo. Se excluyeron ingresos a UCIP por causa no traumática, expediente clínico incompleto, traslados a otro centro hospitalario y pacientes con traumatismo craneoencefálico leve o moderado (Glasgow $\geq 8$ puntos). Se utilizó un instrumento en el cual se registró información sobre datos sociodemográficos, características del traumatismo, manifestaciones clínicas al ingreso, tratamiento en Unidad de Cuidados Intensivos Pediátricos, complicaciones asociadas y condición de egreso. La información recolectada fue ingresada en una base de datos, la cual se diseñó con el paquete estadístico Epiinfo versión 7.2.2.6 (CDC Atlanta), se realizó un análisis univariado. Los resultados se presentan como frecuencias, porcentajes, rangos, promedios de las variables estudiadas. El estudio fue aprobado por las autoridades institucionales. En vista que la fuente de información para la recolección de datos fue una fuente secundaria, no se requirió utilizar consentimiento informado. La información personal de los casos extraída de los expedientes clínicos se manejó de forma confidencial.

\section{RESULTADOS}

El departamento de estadística del HNMCR identificó y proporcionó $77(62.6 \%)$ de los 123 expedientes clínicos solicitados. Los resultados se presentan en base a estos 77 casos.

El grupo etáreo más frecuente fueron los escolares de 6-12 años en un 40.2\% (31), con un promedio de 10 años, predominó el género masculino en $70.1 \%$ (54), la procedencia fue urbana en $64.9 \%$ (50), eran estudiantes $58.4 \%$ (45), con escolaridad básica incompleta en un $37.7 \%$ (29). (Ver Tabla No. 1). 
Tabla No. 1: Distribución de características sociodemográficas de los pacientes pediátricos con TEC Severo atendidos en el HMCR, 2016-2018. N=77.

\section{Características}

Grupo etáreo

Escolar

Adolescente

Preadolescente

Preescolar

Lactante mayor

\section{Edad (años)}

$6-12$

13-14

15-18

3-5

2

Rango

Promedio
N (\%)

31 (40.2)

$16(20.8)$

16 (20.8)

13 (16.9) 1

31 (40.2)

16 (20.8)

16 (20.8)

$13(16.9)$

$1(1.3)$

2-18

10

23 (29.9)

54 (70.1)

50 (64.9)

$27(35.1)$

Rural

45 (58.4)

$28(36.4) 4$

Empleado

Escolaridad

Escolaridad básica incompleta

$29(37.7)$

Escolaridad media incompleta

$16(20.8)$

No aplica

$13(16.8)$

$12(15.6)$

$6(7.8)$

$1(1.3)$

Fuente: Archivo de historias clínicas Hospital Mario Catarino Rivas 2016-2018

El mecanismo de la lesión más frecuente fue el accidente de tránsito en un $64.9 \%$ (50), siendo el lugar de accidente el sitio público en $79.2 \%$ (61), fueron referidos el $89.6 \%$ (69), siendo estos de hospital region- nal en el $72.7 \%$ (56), y siendo el Hospital de El Progreso, quien refirió el $15.6 \%$ (12) y el tipo de accidente el atropellamiento en $36.4 \%$ (28). (Ver Tabla No. 2). 
Tabla No. 2: Características del mecanismo de trauma de los pacientes ingresados a la UCIP por TEC severo atendidos en el HMCR, 2016-2018. N=77.

\section{Características del Trauma}

Mecanismo de la lesión

Accidente de tránsito

Caída en mismo plano de sustentación

Herida por arma blanca
Caída en distinto plano de sustentación

N (\%)

$50(64.9)$

$25(32.4)$

$1(1.3)$

$1(1.3)$

$61(79.2)$

$16(20.8)$

Casa hogar

Referido

$\mathrm{Si}$

$69(89.6)$

No

8 (10.4)

Lugar de referencia

Hospital regional

Cruz Roja/Verde/Bomberos

$56(72.7)$

No aplica

Unidad IHSS

Hospital privado

$8(10.4)$

$6(7.8)$

$3(3.9)$

$3(3.9)$

$1(1.3)$

Unidad De Salud (CIS)

\section{Hospital Regional}

No aplica

Hospital de El Progreso (Yoro)

Hospital Atlántida (Ceiba)

Hosptal Juan Manuel Gálvez (Gracias)

Hospital Regional de Occidente (SRC)

Hospital Puerto Cortés

Hospital San Marcos (Ocotepeque)

Hospital Santa Barbara Integrado (SB)

Hospital San Isidro (Tocoa)

Hospital Tela Integrado (Tela)

$21(27.2)$

$12(15.6)$

$11(14.3)$

9 (11.7)

$6(7.8)$

$5(6.5)$

$5(6.5)$

$4(5.2)$

$2(2.6)$

$2(2.6)$

Transporte de la victima

No aplica

Peatón

26 (33.8)

Motocicleta

$20(26.0)$

Bicicleta

14 (18.2)

7 (9.1)

$5(6.5)$

Automóvil

3 (3.9)

Pickup

$1(1.3)$

Autobús

1 (1.3) 
Tipo de accidente

Atropellamiento

No aplica

Colisión/Choque

Volcamiento

Otro**
$28(36.4)$

$27(35.0)$

$14(18.2)$

$5(6.5)$

$3(3.9)$

* Caballo ** Caída de caballo, bicicleta, paila de automóvil.

Fuente: Archivo de historias clínicas Hospital Mario Catarino Rivas 2016-2018

El 76.6\% (59) presentaron puntaje entre 7-8 según la escala de Glasgow, la región craneal anatómica más afectada fue la temporal en $52.0 \%$ (40), presentando trauma extra-craneano un $85.7 \%$ (66), en miembros superiores e inferiores (54), siendo el tipo de lesión heridas superficial/laceración/excoriación en $54.5 \%$ (42). La manifestación clínica más frecuente fue la alteración del estado de consciencia en un $97.4 \%$ (75), tamaño pupilar $\leq 4 \mathrm{~mm}$ en $68.8 \%$ (53), con presencia de isocoria en un $64.9 \% / 50$ ), con reflejo pupilar presente en el $90.9 \%$ (70), siendo intubados el $100.0 \%$ (77) y presentando pulsioximetría al ingreso $\geq 90 \%$ un 94.8/ (73). (Ver Tabla No. 3).

Tabla No. 3: Manifestaciones clínicas al ingreso de los pacientes pediátricos con TEC Severo atendidos en el HMCR, 2016-2018. $\mathrm{N}=77$.

\begin{tabular}{cc}
\hline Características del Trauma & N (\%) Escala de Glasgow al Ingreso (Puntos) \\
\hline $7-8$ & $59(76.6)$ \\
\hline $4-6$ & $16(20.8)$ \\
3 & $2(2.6)$ \\
Región cráneo afectada & $40(52.0)$ \\
Temporal & $31(40.2)$ \\
Frontal & $24(31.2)$ \\
Parietal & $9(11.7$ \\
Occipital & \\
Presencia de trauma extra-craneano & $66(85.7)$ \\
Si & $11(14.3)$ \\
No & \\
Sitio anatómico afectado & $54(70.1)$ \\
Miembros superiores e inferiores & $23(29.9)$ \\
Cara & $14(18.2)$ \\
Tórax & $11(14.3)$ \\
No aplica & $8(10.4)$ \\
Abdomen & $1(1.3)$ \\
Pelvis & \\
Tipo de lesión & $42(54.5)$ \\
Herida superficial/laceraciones/excoriaciones & $29(37.7)$ \\
Fracturas & $12(15.6)$ \\
Trauma cerrado & $11(23.9)$ \\
No aplica & $10(13.0)$ \\
Otros* &
\end{tabular}


Manifestaciones clínicas

Alteraciones del estado de consciencia

$75(97.4)$

Convulsiones

$22(28.6)$

Vómitos

$22(28.6)$

Hemorragias

Tamaño pupilar

$\leq 4 \mathrm{~mm}$

$53(68.8)$

No Aplica

$20(26.0)$

No consignado

$3(3.9)$

$>4 \mathrm{~mm}$

Alteraciones pupilares

Isocoria

$50(64.9)$

Anisocoria

$23(29.9)$

Miosis

Midriasis

\section{Reflejo pupilar}

Presente

$70(90.9)$

Ausente

Signos clínicos de lesiones graves

Ninguno

$55(71.4)$

Epistaxis

$12(15.6)$

Otorragia/hemotímpano

$7(9.1)$

Equimosis periorbitaria

$6(7.8)$

Signo de battle (equimosis retroauricular)

Fue intubado

Si

77 (100.0)

No

$0(0.0)$

Pulsioximetría al ingreso

$\geq 90 \%$

$73(94.8)$

$<90 \%$

$4(5.2)$

*Otros: Amputación traumática de mano (1), contusión pulmonar (5), lesión esplénica (1), neumotórax (2), tórax inestable (1).

Fuente: Archivo de historias clínicas Hospital Mario Catarino Rivas 2016-2018

Se encontró normoglicemia en el 88.3\% (68), niveles de sodio entre 135-145 mEq/L en $77.9 \%$ (60), hemoglobina $\geq 8$ en $96.1 \%$ (74), plaquetas $\geq 75,000$ en $98.7 \%$ (76) e INR < 1.4 en $59.7 \%$ (46). Se realizó TAC en $96.1 \%$ (74), siendo el principal hallazgo imagenológico el edema cerebral en $79.2 \%$ (61). No ameritó neurocirugía el 70.1\% (54). Se manejó la hiperglicemia en $61.0 \%$ (47), se brindó terapia hiperosmolar con SSN 3\% en $90.9 \%$ (70), ameritó transfusión sanguínea $62.3 \%$ (48), se utilizó aminas vasoactivas en $63.6 \%$ (49), con manejo para el dolor de tipo sedo-analgesia en infusión en $62.3 \%$ (48), iniciando terapia nutricional enteral s 12 horas en $45.9 \%$ (34), colocándose catéter venoso central en $97.4 \%$ (75). (Ver Tabla No. 4, 5). 
Tabla No. 4: Datos laboratoriales e imagenológicos de los pacientes pediátricos con TEC Severo atendidos en el HMCR, 2016-2018. N=77.

\begin{tabular}{|c|c|}
\hline Exámen & $\mathbf{N}(\%)$ \\
\hline \multicolumn{2}{|l|}{ Se realizó TAC } \\
\hline $\mathrm{Si}$ & $74(96.1)$ \\
\hline No & $3(3.9)$ \\
\hline \multicolumn{2}{|l|}{ Hallazgos de TAC } \\
\hline Edema cerebral & $61(79.2)$ \\
\hline Contusión hemorrágica & $19(24.7)$ \\
\hline Hemorragia subaracnoidea & $17(22.1)$ \\
\hline Hematoma epidural & $14(18.2)$ \\
\hline Fractura NO desplazada & $11(14.3)$ \\
\hline Hematoma subdural & $9(11.7)$ \\
\hline Fractura de cráneo desplazada & $6(7.8)$ \\
\hline Hemorragia subgaleal & $3(3.9)$ \\
\hline Hemorragia intracraneal & $2(2.6)$ \\
\hline Desviación de la línea media & $1(1.3)$ \\
\hline Hemorragia intraventricular & $1(1.3)$ \\
\hline Otros* & $2(2.6)$ \\
\hline \multicolumn{2}{|l|}{ Ameritó neurocirugía } \\
\hline No & $54(70.1)$ \\
\hline $\mathrm{Si}^{* *}$ & $23(29.9)$ \\
\hline \multicolumn{2}{|l|}{$\begin{array}{l}\text { Valores laboratoriales al Ingreso } \\
\text { Glicemia (mg/dl) }\end{array}$} \\
\hline $\begin{array}{l}80-180 \\
>180 \\
<80\end{array}$ & $\begin{array}{l}68(88.3) \\
5(6.5) \\
4(5.2)\end{array}$ \\
\hline \multicolumn{2}{|l|}{ Sodio (mEq/L) } \\
\hline $135-145$ & $60(77.9)$ \\
\hline $146-160$ & $8(10.4)$ \\
\hline$>160$ & $5(6.5)$ \\
\hline$<135$ & $4(5.2)$ \\
\hline \multicolumn{2}{|l|}{ Hemoglobina ( $/ \mathrm{dl})$} \\
\hline $\begin{array}{l}\geq 8 \\
<8\end{array}$ & $\begin{array}{c}74(96.1) \\
3(3.9)\end{array}$ \\
\hline \multicolumn{2}{|l|}{ Plaquetas $\left(\mathrm{mm}^{3} / \mathrm{ml}\right)$} \\
\hline$\geq 75,000$ & $\begin{aligned} 76 \\
1(13)\end{aligned}$ \\
\hline$<75,000$ & \\
\hline $\begin{array}{l}\text { INR } \\
<1.4\end{array}$ & INR \\
\hline$\geq 1.4$ & $31(40.3)$ \\
\hline
\end{tabular}

*Hidrocefalia, neumoencéfalo neumoventrículo.

**Indicación: Colocación DVP (1), cráneo hipertensivo (1), craneotomía descompresiva (7), drenaje hematoma epidural (6), drenaje hematoma subdural (3), fractura con hundimiento (1), fractura expuesta de cráneo (1), síndrome de herniación uncal (1), no especificado (2)

Fuente: Archivo de historias clínicas Hospital Mario Catarino Rivas 2016-2018 
Tabla No. 5: Manejo terapéutico dentro de la UCIP de los pacientes pediátricos con TEC Severo atendidos en el HMCR, 2016-2018. $\mathrm{N}=77$.

\section{Medida Terapéutica}

Manejo de hiperglicemia

No

$\mathrm{Si}$

Tiempo (10-48 hrs)

Terapia hiperosmolar

Solución salina hiperosmolar (3\%)

Ninguno

Transfusión sanguínea

$\mathrm{Si}$

No

Tipo de derivado sanguíneo

GRE (1-3 unidades)

Plasma (13 unidades)

Presión arterial media $\geq$ percentil 50 para edad

No

$\mathrm{Si}$

Uso de aminas vasoactivas Si 49
No

(63.6)

Tipo de aminas vasoactivas utilizadas

Epinefrina

Norepinefrina

Dopamina

Dobutamina

Temperatura al ingreso

36.5-38.3

$<36.5$

$>38.3$

Valores de $\mathrm{PaCO}^{2}(\mathrm{mmHg})$ al ingreso:

35-45

$<35$

$>45$

Uso de antibióticos intravenosos:

$\mathrm{Si}$

Manejo del dolor:

Sedo-analgesia en infusión

Sedación en infusión

Analgésicos a horario

Tipo de analgésicos

Fentanilo/Midazolam

Midazolam

Terapia nutricional:

Si (Enteral)

No se inicio
N (\%)

$47(61.0)$

$15(19.5)$

$15(19.5)$

$70(90.9)$

$7(9.1)$

$48(62.3)$

29 (37.7)

45 (58.4)

$14(18.2)$

46 (59.7)

$31(40.3)$

28 (36.4)

33 (42.8)

27 (35.1)

15 (19.5)

63 (81.8)

$7(9.1)$

$7(9.1)$

73 (94.8)

$3(3.9)$

$1(1.3)$

77 (100.0)

48 (62.3)

$29(37.7)$

$0(0.0)$

$50(65.0)$

$27(35.0)$

74 (96.1)

3 (3.9) 
Tiempo de inicio nutrición enteral:

$\leq 12$ horas

13-47 horas

$34(45.9)$

48-72 horas

Terapia profiláctica anticonvulsivante

$\mathrm{Si}$

$29(39.1)$

$11(15.0)$

No

Cabeza central y elevada a $30^{\circ}$

$\mathrm{Si}$

Se colocó catéter venoso central

$\mathrm{Si}$

No

$51(66.2)$

$26(33.8)$

Tipo de acceso venoso

$77(100.0)$

Subclavio derecho

Subclavio izquierdo

$75(97.4)$

$2(2.6)$

No aplica

Fuente: Archivo de historias clínicas Hospital Mario Catarino Rivas 2016-2018

La complicación más frecuente en UCIP fue la neumonía asociada a ventilación mecánica en el 30\% (Ver gráfico1), el tiempo de permanencia en la UCIP fue de $48 \mathrm{hrs}$ a 1 semana en el $48 \%$ (Ver Gráfico No. 2), la tasa de mortalidad fue el $10.0 \%(8 / 77)$ (Ver Gráfico No. 3), la sala hospitalaria con mayores traslados fue neurocirugía pediátrica en $45.5 \%$ (Ver Gráfico No. 4) respectivamente. 
Gráfico No. 1: Complicaciones presentadas en los pacientes con TEC Severo ingresados a UCIP 2016-2018, N=77



*Otras complicaciones: Daño axonal difuso (2), ECV isquémico (1), traqueostomía (3), gastrostomía (2), Neumotórax (3), úlceras corneales (1) ** SDRAS: Síndrome de Distrés Respiratorio Agudo / CID: Coagulación Vascular / IAAS: Infecciones asociadas a atención en salud.

Fuente: Archivo de historias clínicas Hospital Mario Catarino Rivas 2016-2018

Gráfico No. 2: Tiempo de permanencia hospitalaria en UCIP de los pacientes pediátricos con TEC severo, 2016-2018. $\mathrm{N}=77$.

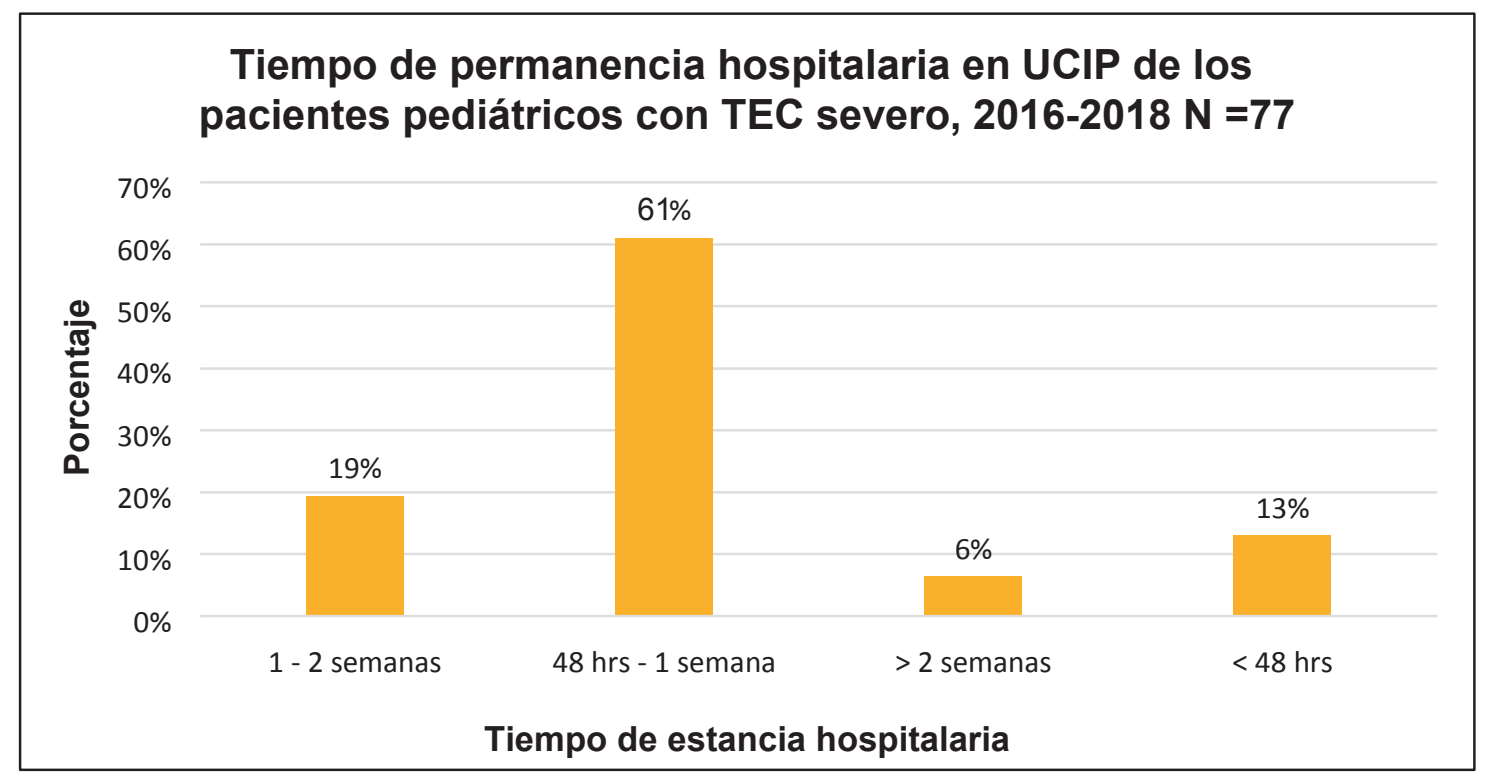

Fuente: Archivo de historias clínicas Hospital Mario Catarino Rivas 2016-2018 
Gráfico No. 3: Condición de egreso en UCIP de los pacientes pediátricos con TEC severo, 2016-2018. $\mathrm{N}=77$.

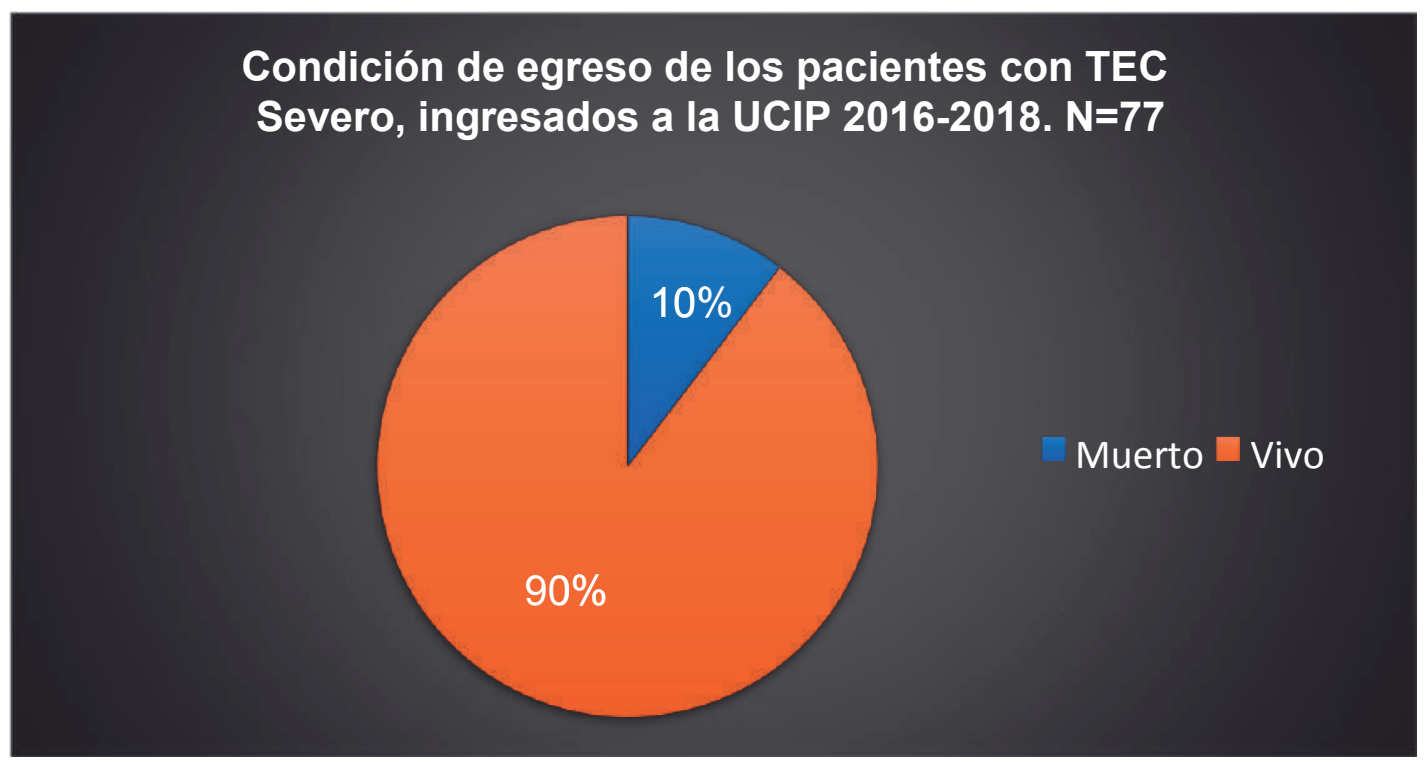

Fuente: Archivo de historias clínicas Hospital Mario Catarino Rivas 2016-2018

Gráfico No. 4: Traslado a sala hospitalaria de los pacientes pediátricos con TEC severo, 2016-2018. $\mathrm{N}=77$.



Fuente: Archivo de historias clínicas Hospital Mario Catarino Rivas 2016-2018 


\section{DISCUSIÓN}

El traumatismo craneoencefálico es una condición común que afecta a niños de todo el mundo y representa un problema de salud pública mundial y es completamente subestimado en nuestro país debido a la falta de investigación y datos de buena calidad, solo en los Estados Unidos se estima que 475,000 niños de 0 a 14 años sufren cada año 1 traumatismo craneoencefálico. Esto resulta en más de 7,000 muertes, 60,000 hospitalizaciones y 600,000 visitas al departamento de emergencia anualmente entre niños estadounidenses. ${ }^{(15,16)}$ La edad media de incidencia en el presente estudio fue de 10 años, contrario a las edades encontradas en literaturas consultadas como el estudio realizado por Corea Galeano X. y colaboradores en Nicaragua que fue de 5.27 años, ${ }^{(17)}$ y el estudio de Chaitanya K. y colaboradores en la India que fue de 5.5 años. ${ }^{(18)}$

Los datos emitidos por el CDC (Centers for Disease Control and Prevention) en su reporte de distribución porcentual de las lesiones relacionadas con TCE por grupo de edad y mecanismo de lesión en Estados Unidos (2006-2010) describen que $72.8 \%$ de los niños de 0-4 años acudieron a urgencias por caídas de altura como principal etiología del TCE y que las contusiones, lesiones y accidentes de tránsito por vehículos de motor fueron las causas más frecuentes de TCE en niños de 5 a los 14 años de edad y de 15 a 24 años. ${ }^{(18,19)}$ La causa principal de TCE que se encontró en este estudio fue el accidente de tránsito en escolares, preadolescentes y adolescentes y la segunda causa las caídas de altura mostrando similitud en el mecanismo de trauma según la población por edad afectada según este reporte. Lo que muestra relevancia es, que, en comparación con los niños más pequeños, los niños mayores tienen una mayor incidencia de lesiones moderadas a graves que son tratadas en el hospital y una incidencia mucho mayor de lesiones fatales. ${ }^{(20)}$ Se identificó como mecanismo del trauma la caída de caballo, lo que constituye una similitud con el estudio nicaragüense ya que acá igualmente aún se observa transitar por las calles carretas de caballos y aun se transportan en algunas áreas de esta manera.

El sexo masculino predomino con una relación de 4 hombres por cada mujer (4:1) acorde a lo reportado en diferentes estudios donde los pacientes del sexo masculino son los más afectados con diferentes relaciones descritas siendo la menor 1.05:1 reportando la mayoría $1.8: 1 .^{(16,18)}$ El $64.9 \%$ de los pacientes provenían de áreas urbanas, áreas donde se encuentra mayor circulación vehicular y que corresponde al principal mecanismo de trauma encontrado.

El $89.6 \%$ de los pacientes eran referidos encontrando como desventaja que los lugares de referencia al HNMCR se encuentran a más de una hora de distancia, la llegada tardía de los pacientes del sitio de la lesión y/o del hospital primario de referencia aumenta su morbilidad al no poder recibir el manejo inicial en "la hora dorada", lo que no es acorde al estudio nicaragüense realizado por Corea Galeano X. y colaboradores ${ }^{(17)}$ donde describen que al igual que este estudio sus pacientes provienen de áreas rurales pero mejor tiempo de transporte para estos y mejores condiciones descritas de los hospitales primarios que refieren, considerando que los centros regionales de nuestro país no cuentan con tomografía axial computarizada entre otras necesidades.

La tasa de mortalidad que se encontró en el estudio fue de $10 \%$ en comparación a la del estudio nicaragüense donde apenas alcanzan el $4.3 \%$; iguales tasas de mortalidad a la nuestra se observan reportadas para áreas 
de Sudáfrica, España, Suecia y Estados Unidos en el estudio de Revisión de la Epidemiologia Global del TEC de Dewan M.C. y colaboradores del año 2016.(16)

El síntoma que con mayor frecuencia se encontró descrito fue la pérdida del estado de conciencia en el $97.4 \%$ de los pacientes, seguido de vómito y convulsiones en $28.6 \%$, los niños con vómitos como síntoma de presentación única generalmente no tienen lesión intracraneal, pero el antecedente de vómitos después de un traumatismo de cráneo aumenta los riesgos posteriores. A diferencia de otros estudios donde engloban el TEC leve, moderado y severo dentro de sus muestras poblacionales y que el vómito es el síntoma encontrado con mayor frecuencia este estudio engloba solamente pacientes con traumatismo de cráneo severo lo que podría considerarse como causa para obtener diferentes resultados. ${ }^{(18,21)}$

La neuroimagen desempeña un papel crítico en la evaluación de pacientes con TCE, actualmente, el objetivo principal de la obtención de imágenes en TCE es determinar la presencia o ausencia de lesión cerebral clínicamente abordable ya sea de manera quirúrgica, ingreso con observación cercana o alta en el hogar. Existe un fuerte consenso y evidencia de que la Tomografía Computarizada sin contraste es la prueba de imagen diagnóstica inicial elegida para los pacientes con TCE agudo de moderada a severa (recomendación de clase I). ${ }^{(22)}$

Se encontró que solo el $96.1 \%$ de los pacientes contaban con estudio de tomografía computarizada resultando que a tres de los pacientes no se realizó el estudio. El edema cerebral fue el hallazgo más frecuente en los estudios de TC realizados, seguido de la contusión hemorrágica al igual que el estudio de Corea Galeano X. y colaboradores en Nicaragua y al de Satapathy M. y colaboradores realizado en la India en el año 2016, contrario al de Chaitanya K. y colaboradores en la India del 2018 en el que el hallazgo tomográfico más frecuente fue la fractura de cráneo. ${ }^{(17,18,23)}$

Se evidenció que el $29.9 \%$ de los pacientes requirió manejo neuroquirúrgico contrario al $19.7 \%$ y $10 \%$ reportado por Satapathy $M$. en el 2016 y Chaitanya en el 2018 en la india respectivamente y similar al $17.4 \%$ reportado en el estudio nicaragüense de Corea Galeano X. y colaboradores considerando que los pacientes que reportan con Glasgow menor de 9 solo corresponden a 2 pacientes y 8 que no se les realizo valoración de Glasgow por estar bajo efecto de sedación según describen en el estudio al ingreso a su hospital. ${ }^{(17,18,23)}$

Las técnicas de monitoreo en pacientes con lesión cerebral traumática se pueden dividir en tres categorías: monitoreo de presión intracraneal, monitoreo de flujo sanguíneo y monitoreo de sustratos bioquímicos. En nuestro centro hospitalario no se cuenta con tecnología para la medida de la PIC, ni del flujo sanguíneo cerebral lo cual está indicado dentro del manejo, sobre todo en aquellos pacientes sometidos a procedimientos neuroquirúrgicos. A pesar de no contar con tecnología de punta, la clínica continúa siendo el pilar más importante del tratamiento en este tipo de pacientes. El advenimiento de la formulación de guías internacionales de manejo permite el manejo dinámico de los que sufren TEC severo. ${ }^{(8,9,24,25)}$

La piedra angular del tratamiento para el TCE severo es la prevención / mitigación de una lesión cerebral secundaria relacionada principalmente con la perfusión cerebral inadecuada que resulta del desarrollo de 
hipertensión intracraneal, la perfusión cerebral inadecuada o el aumento del metabolismo cerebral. En el estudio publicado por la Revista Critical Care en el 2014 sobre si la adherencia a las pautas de la guía del 2003 mejoraba la supervivencia y el pronóstico neurológico se evidencio que tres recomendaciones de la guía se asociaron de forma independiente con la supervivencia: ausencia de hipoxia prehospitalaria, inicio temprano de nutrición (antes de las primeras 72 horas) y $\mathrm{PaCO} 2>30 \mathrm{~mm} \mathrm{Hg}$ en ausencia de signos radiológicos o clínicos de hernia cerebral. ${ }^{(24,26)}$

Dentro de nuestros pacientes se documentó el apego a dichas recomendaciones encontrando que el $96.1 \%$ de los pacientes inicio nutrición enteral temprana antes de las 72 horas y de estos 34 (45.9\%) de los pacientes la iniciaron antes de las 12 horas, encontrando estudios similares como el de Ponce y Ponce de León del año 2015 donde se describe que el inicio de la nutrición enteral temprana antes de las 24 horas disminuye las comorbilidades asociadas a la desnutrición intrahospitalaria, así como días de estancia hospitalaria y días de ventilación mecánico. ${ }^{(26)}$

Los pacientes en los que se evidencio datos de edema cerebral recibieron manejo con solución salina hipertónica al 3\%, se evitó desde su ingreso a la unidad datos de hiperventilación o de hipoxia, con saturaciones de pulsioximetria, niveles de $\mathrm{PaCO} 2$ adecuados al ingreso a la unidad; así como también se evidencio el seguimiento de recomendaciones que permiten disminuir la tasa metabólica cerebral manteniendo la eutermia, niveles de glicemia, evitando el dolor, las convulsiones y mantener los valores laboratoriales dentro de lo recomendado en cuanto a valor plaquetario, INR y hemoglobina.

En los 8 pacientes que fallecieron se evidencio múltiples factores de mal pronóstico a su ingreso dentro de los que podríamos destacar que $6(75 \%)$ tenían Glasgow de 6 a 5 y un pacientes de 3 a 4 puntos, el $75 \%$ tenían reflejo pupilar ausente, 5 (62.5\%) requirieron neurocirugía y 4 ingresaron con valores de sodio menores a los recomendados por la guía de manejo y uno solo manifestó hipernatremia con sodio mayor a $160 \mathrm{mEq} / \mathrm{L}$ a las 24 horas de su estancia. Además 3 de los pacientes requirieron larga estadía hospitalaria e inicio tardío de alimentación enteral relacionándose con la presencia de enfermedades propias de la UCIP como complicación y aumento de su morbilidad. $(8,9,27,28)$

Conflictos de interés: Los autores declaran que no existe conflicto de interés en la publicación del presente artículo. 


\section{REFERENCIAS BIBLIOGRÁFICAS}

1. Marín González A.L. TRAUMA EN PEDIATRÍA. Rev Mex Anest. 2017; 40 (Supl 1 abril - junio): pp S52-S54.

Disponible en: http://www.medigraphic. com/pdfs/rma/cma-2017/cmas171s.pdf.

2. Bustos Córdova E, Cabrales Martínez R. G, Cerón Rodríguez M, Naranjo López M.Y. EPIDEMIOLOGÍA DE LESIONES NO INTENCIONALES EN NIÑOS: REVISIÓN DE ESTADÍSTICAS INTERNACIONALES Y NACIONALES. Bol Med Hosp Infant Mex. 2014; 71(2):68-75.

Disponible en: http://www.scielo.org.mx/ $\mathrm{pdf} / \mathrm{bmim} / \mathrm{v} 71 \mathrm{n} 2 / \mathrm{v} 71 \mathrm{n} 2 \mathrm{a} 2$.pdf.

3. Thurman DJ. The Epidemiology of Traumatic Brain Injury in Children and Youths: A Review od Research Since 1990. J Child Neurol. 2016;31(1):20-7.

4. Lozano Gonzales D.A, Ayala Méndez L.R. INTEGRACIÓN SENSORIAL EN NEURORREHABILITACIÓN CON PACIENTES DE TRAUMA CRANEOÉNCEFALICO (TCE) EN UCI PEDIÁTRICA. fisioGlía 2018, 5(2): 35-41.

Disponible en: https://dialnet.unirioja.es /servlet/articulo?codigo $=6397988$.

5. De la Torre-Gómez R.E, Rodríguez-Rodríguez I.C, López-León A, Carranza-Barrera L.G, Brancaccio-Orozco J, Guzmán-Rodríguez I, Et al. Revisión de trauma de cráneo severo en niños. Rev Med MD. 2014 5(4):229-237pp.

6. Casas Fernández C. Traumatismos craneoencefálicos. Protocolos Diagnóstico Terapéuticos de la AEP: Neurología Pediátrica. Cap.17, 2da edición, 2008.
Pág. 118-128.

Disponible en: http:// www.aeped.es/sites/default/files/documentos/17- tce.pdf.

7. Matamoros D.J, Zúñiga D.B, Tomé E. Aspectos Epidemiológicos del trauma en pacientes pediátricos ingresados en la emergencia pediátrica del Hospital escuela y del Instituto Hondureño de seguridad social revista médica de los postgrados de medicina UNAH vol.10 no.1 enero-abril 2007. Disponible en: http://cidbimena.desastres.hn/RMP/ pdf/2007/html/Vol10-S-2007.html.

8. Carney N, Totten AM, O'Reilly C, Ullman JS, Hawryluk GW, Bell MJ. Et al. Guidelines for the Management of Severe Traumatic Brain Injury, Fourth Edition. Nuerosurgery. 2017;1(80):6-15.

Disponible en: doi:10.1227/NEU.0000000000001432.

9. Kochanek P.M, Carney N, Adelson P.D, Ashwal S, Bell M.J, Bratton S. Et al. Guidelines for the acute medical Management of Severe Traumatic Brain Injury in Infants, Children and Adolescents-Second Edition. Pediatr Crit Care Med. 2012; 13(1 Suppl.): S1-S82. Disponible en: https://braintrauma.org/uploads/03/ 15/guidelines_pediatric2_2.pdf.

10. Silva Higuero N, García Ruano A. Traumatismos Craneoencefálicos. Pediatr Integral 2014; XVIII(4): 207-218.

11. Orliaguet G, Uhrig L. Traumatismo craneoencefálico del niño. EMC - Anestesia-Reanimación. 2016; 42(4): 1-15. Archivo disponible en: http://dx.doi.org/ 10.1016/S1280-4703(16)80724-0. 
12. Hardcastle N, Benzon H.A, Vavilala M.S. Update on the 2012 guidelines for the management of pediatric traumatic brain injury - information for the anesthesiologist. Paediatr Anaesth. 2014; 24(7): 703-710.

Disponible en: doi:10.1111/pan.12415.

13. Oliva Meza Hernández O.M, Maya Bautista D.K. Traumatismo Craneoencefálico Grave en Pediatría. An Med (Mex) 2016; 61 (4): 261-270.

14. Madrigal Ramírez E, Hernández Calderón C. Generalidades de Trauma Cráneo Encefálico en Medicina Legal. Med Leg de Costa Rica. [Internet] 2017 a [consultado 02 de enero 2018]; 34(1). Disponible en: http://www.scielo.sa.cr/ pdf/mlcr/v34n1/1409-0015-mlcr-34-01 -00147.pdf.

15. García-Lira J.R, Zapata-Vázquez R.E, Alonzo-Vázquez F, Rodríguez-Ruz S.G Medina-Moreno M.R. Torres-Escalante J.L. Monitorización de la presión intracraneal en traumatismo craneoencefálico severo. Rev Chil Pediatr. 2016; 87(5):387-394.

16. A Dewan MC, Mummareddy N, Wellons III JC, Bonfield CM, The epidemiology of global pediatric traumatic brain injury: a qualitative review, World Neurosurgery (2016), doi:10.1016/j.wneu.2016. 03.045.

17. Galeano Corea X, Hernández R, Gómez RJ. Manejo y evolución de pacientes con traumatismo craneoencefálico severo en las Unidades de Terapia Intensiva del Hospital Infantil de Nicaragua "Manuel de Jesús Rivera". Nicaragua Pediatr @2014;2(2):18-21.
18. Chaitanya K, Addanki A, Karambelkar R, Ranjan R. Traumatic brain injury in Indian children. Childs Nerv Syst. 2018 Jun; 34(6):1119-1123.

Doi: https://doi.org/10.1007/s00381-018 -3784-z.

19. Avilés-Martínez KI, Cruz-López PJM, García-Armenta B, Jiménez-Pérez BA, López-Enríquez A, Montaño-Dorado CJ. Perspectiva del trauma craneoencefálico en urgencias de pediatría. Rev Mex Pediatr 2015; 82(4):129-134.

20. Thurman DJ. The Epidemiology of Traumatic Brain Injury in Children and Youths: A Review of Research Since 1990. Journal of Child Neurology 2016, Vol. 31(1) 20-27. DOI: $10.1177 / 0883073814544363$.

21. Dayan PS, Holmes JF, Atabaki S, et al. Association of traumatic brain injuries with vomiting in children with blunt head trauma. Ann Emerg Med. 2014; 63(6): 657-665;

doi:10.1016/j.annemergmed.2014.01. 009 .

22. Wintermark M, Sanelli PC, Anzai $Y$, Tsiouris A.J, Whitlow CT. Imaging Evidence and Recommendations for Traumatic Brain Injury: Conventional Neuroimaging TechniquesJ Am Coll Radiol 2015;12:e1-e14. DOI: http:// dx. doi.org/10.1016/j.jacr.2014.10.014.

23. Satapathy M, Dash D, Mishra S, Tripathy S, Nath P, Jena S (2016) Spectrum and outcome of traumatic brain injury in children $<15$ years: a tertiary level experience in India. Int J Crit IIIn Injury Sci 6(1):16-17. doi: 10.4103/2229-5151.17 7359 . 
24. Vavilala MS, Kernic MA, Wang J, et al for the Pediatric Guideline Adherence and Outcomes Study. Acute care clinical indicators associated with discharge outcomes in children with severe traumatic brain injury. Crit Care Med. 2014; 42(10):2258-2266;

doi:10.1097/CCM.0000000000000507.

25. Godoy DA, Lubillo S, Rabinstein AA. Pathophysiology and Management of Intracranial Hypertension and Tissular Brain Hypoxia After Severe Traumatic Brain Injury An Integrative Approach. Neurosurg Clin N Am 29 (2018) 195-212. DOI: https://doi.org/10.1016/ j.nec.2017. 12.001.

26. Ponce y Ponce de León, G, Mayagoitia Witrón, JdJ, Cornejo Bravo, JM, Pérez Morales, ME. Impacto de la nutrición enteral temprana en pacientes con trau- matismo craneoencefálico en la Unidad de Cuidados Intensivos de un hospital mexicano. RIDE Revista Iberoamericana para la Investigación y el Desarrollo Educativo [Internet]. 2015;6(11). Recuperado de: https://www.redalyc.org/ar ticulo.oa? id=498150319042.

27. Moscote-Salazar LR, Rubiano AM, Alvis-Miranda HR, Calderon-Miranda W, Alcala-Cerra G, Rivera MAB, Agrawal A. Severe Cranioencephalic Trauma: Prehospital Care, Surgical Management and Multimodal Monitoring. Bull Emerg Trauma. 2016;4(1):8-23.

28. Stocchetti N, Carbonara M, Citerio G, Ercole A, Skrifvars MB, Smielewski P. et al. Severe traumatic brain injury: targeted management in the intensive care unit. Lancet Neurol 2017; 16: 452-64. 